\title{
ON THE IRREDUCIBILITY OF LINEAR REPRESENTATIONS OF THE PURE BRAID GROUP
}

\author{
MOHAMMAD N. ABDULRAHIM
}

\begin{abstract}
Following up on our result in [1], we find a milder sufficient condition for the tensor product of specializations of the reduced Gassner representation of the pure braid group to be irreducible. We prove that $G_{n}\left(x_{1}, \ldots, x_{n}\right) \otimes G_{n}\left(y_{1}, \ldots, y_{n}\right)$ : $P_{n} \rightarrow G L\left(\mathbb{C}^{n-1} \otimes \mathbb{C}^{n-1}\right)$ is irreducible if $x_{i} \neq \pm y_{i}$ and $x_{j} \neq \pm y_{j}{ }^{-1}$ for some $i$ and $j$.
\end{abstract}

\section{Introduction}

The pure braid group, $P_{n}$, is a normal subgroup of the braid group, $B_{n}$, on $n$ strings. It has a lot of linear representations. One of them is the Gassner representation which comes from the embedding $P_{n} \rightarrow \operatorname{Aut}\left(F_{n}\right)$, by means of Magnus representation [3, p.119]. In our work, we consider the tensor product of complex specializations of irreducible Gassner representation of the pure braid group, namely,

$$
G_{n}\left(x_{1}, \ldots, x_{n}\right) \otimes G_{n}\left(y_{1}, \ldots, y_{n}\right): P_{n} \rightarrow G L\left(\mathbb{C}^{n-1} \otimes \mathbb{C}^{n-1}\right) .
$$

Our previous work in [1] asserts that for $n \geq 3$ and $x_{1}, \ldots, x_{n}, y_{1}, \ldots, y_{n} \in \mathbb{C}-\{0,1\}$, the representation above is irreducible under the following condition: for some $i$ and $j \in$ $\{1, \ldots, n\}, \quad i<j, \quad x_{i} x_{j} \neq y_{i} y_{j} \quad$ and $\quad x_{\alpha} x_{j} y_{\alpha} y_{j} \neq 1$, for every $\alpha \in\{1, \ldots, j-$ $1\}$ and $x_{\alpha+1} x_{j} y_{\alpha+1} y_{j} \neq 1$, for every $\alpha \in\{j, \ldots, n-1\}$.

In Section 1 of our work, We define the Gassner representation of a free normal subgroup of the pure braid group of rank $n-1$ denoted by $U_{r}$ where $1 \leq r \leq n$. We consider $\mathbb{C}\left[U_{r}\right]$ to be the group algebra of $U_{r}$ over $\mathbb{C}$, and let $\mathcal{A}$ be the augmentation ideal of $\mathbb{C}\left[U_{r}\right]$. On the other hand, if $M$ is any $P_{n}$-module, then $\mathcal{A} M$ is a $P_{n}$-submodule of $M$. We first show that if $\mathbb{C}^{n-1}$ is made into a $P_{n}$-module via the specialization of the reduced Gassner representation $G_{n}\left(x_{1}, \ldots, x_{n}\right): P_{n} \rightarrow G L\left(\mathbb{C}^{n-1}\right)$, then $\mathcal{A} \mathbb{C}^{n-1}$ is its unique minimal nonzero $P_{n}$-submodule. Of course $\mathcal{A} \mathbb{C}^{n-1}=\mathbb{C}^{n-1}$ when $G_{n}\left(x_{1}, \ldots, x_{n}\right)$ is irreducible.

In Section 2, we give a summary of the proofs of the important results published in [1], which will help us to prove our main theorem.

Received September 18, 2009.

2000 Mathematics Subject Classification. Primary: 20F36.

Key words and phrases. Braid group, pure braid group, Gassner representation. 
In Section 3 , we let $x_{1}, \ldots, x_{n}, y_{1}, \ldots, y_{n} \in \mathbb{C}-\{0\}$, so that $G_{n}\left(x_{1}, \ldots, x_{n}\right) \otimes G_{n}\left(y_{1}, \ldots\right.$, $y_{n}$ ) defines a diagonal action of $P_{n}$ on $\mathbb{C}^{n-1} \otimes \mathbb{C}^{n-1}$. The main technical result is Proposition 1, which gives a sufficient condition for $\mathcal{A} \mathbb{C}^{n-1} \otimes \mathcal{A} \mathbb{C}^{n-1}$ to be the unique minimal nonzero $P_{n}$-submodule of $\mathbb{C}^{n-1} \otimes \mathbb{C}^{n-1}$. This implies the irreducibility of the tensor product above. We then state Lemma 4 to simplify Proposition 1 and prove our main theorem that states the following: If $x_{i} \neq \pm y_{i}$ and $x_{j} \neq \pm y_{j}{ }^{-1}$ for some $i$ and $j \in\{1, \ldots, n\}$ then the tensor product above is irreducible.

Notation 1. The pure braid group, $P_{n}$, is defined as the kernel of the homomorphism $B_{n} \rightarrow S_{n}$, defined by $\sigma_{i} \rightarrow(i, i+1), 1 \leq i \leq n-1$. It has the following generators:

$$
A_{i, r}=\sigma_{r-1} \sigma_{r-2} \ldots \sigma_{i+1} \sigma_{i}{ }^{2} \sigma_{i+1}^{-1} \ldots \sigma_{r-2}^{-1} \sigma_{r-1}^{-1}, \quad 1 \leq i<r \leq n
$$

We will construct for each $r=1, \ldots, n$ a free normal subgroup of rank $n-1$, namely, $U_{r}$. Let $U_{r}$ be the subgroup generated by the elements

$$
A_{1, r}, A_{2, r}, \ldots, A_{r-1, r}, A_{r, r+1}, \ldots, A_{r, n},
$$

where $A_{i, r}$ are those generators of $P_{n}$ that become trivial after the deletion of the $r$-th strand. For a fixed value of $r$, the image of $A_{i r}$ under the reduced Gassner representation is denoted by $\tau_{i, r}$, where $\tau_{i, r}=I-P_{i, r} Q_{i, r}$. In other words, the generators of $U_{r}$ are $A_{i, r}$ where $A_{i, r}=A_{r, i}$ whenever $i>r$. It is known that $U_{r}$ generates a free subgroup of $P_{n}$ which is isomorphic to the subgroup $U_{n}$ freely generated by $\left\{A_{1, n}, A_{2, n}, \ldots, A_{n-1, n}\right\}$. This is intuitively clear because it is quite arbitrary how we assign indices to the braid "strings".

For simplicity, we denote $A_{i, r}$ by $\tau_{i, r}$. That is, we have

$$
\tau_{1, r}=A_{1, r}, \ldots, \tau_{r-1, r}=A_{r-1, r}, \quad \tau_{r+1, r}=A_{r, r+1}, \quad \tau_{r+2, r}=A_{r, r+2}, \ldots, \tau_{n, r}=A_{r, n}
$$

Definition 1. The reduced Gassner representation restricted to $U_{r}$ is defined as follows: $\tau_{i, r}=I-P_{i, r} Q_{i, r}$ for $1 \leq i, r \leq n$. For $i<r, P_{i, r}$ is the column vector given by

$$
(1-t_{1}, \ldots, 1-t_{i-1}, \underbrace{1-t_{i} t_{r}}_{i}, t_{r}\left(1-t_{i+1}\right), \ldots, t_{r}\left(1-t_{r-1}\right), \underbrace{t_{r+1}-1}_{r}, t_{r+2}-1, \ldots, t_{n}-1)^{T},
$$

and for $n \geq i>r, P_{i, r}$ is the column vector given by

$(t_{r}\left(t_{1}-1\right), \ldots, t_{r}\left(t_{r-1}-1\right), \underbrace{1-t_{r+1}, \ldots, 1-t_{i}}_{i-r}, 1-t_{i+1} t_{r}, t_{r}\left(1-t_{i+2}\right), \ldots, t_{r}\left(1-t_{n}\right))^{T}$.

Here $T$ is the transpose and $Q_{i, r}$ is the row vector given by

$$
Q_{i, r}=(0, \ldots, 0, \underbrace{1}_{i}, 0, \ldots, 0), \quad 1 \leq i, r \leq n .
$$


The definition of the reduced Gassner representation restricted to a free normal subgroup is the same, up to equivalence, as the definition in [3]. Representations given by pseudoreflections $I-A_{i} B_{i}$ and $I-C_{i} D_{i}$ are equivalent if the inner products $\left(B_{i} A_{j}\right)$ and $\left(D_{i} C_{j}\right)$ are conjugate by a diagonal matrix. Here, $A_{i}, C_{i}$ are column vectors and $B_{i}, D_{i}$ are row vectors. For more details, see [4].

We identify $\mathbb{C}^{n-1}$ with $(n-1) \times 1$ column vectors. We let $e_{1}, \ldots, e_{n-1}$ denote the standard basis for $\mathbb{C}^{n-1}$, and we consider matrices to act by left multiplication on column vectors.

Definition 2. If $r=a_{1} e_{1}+\cdots+a_{n-1} e_{n-1} \in \mathbb{C}^{n-1}$, the support of $r$, denoted supp $(r)$, is the set $\left\{e_{i} \mid a_{i} \neq 0\right\}$. If $s=\Sigma a_{i j}\left(e_{i} \otimes e_{j}\right) \in \mathbb{C}^{n-1} \otimes \mathbb{C}^{n-1}$, the support of $s$, also denoted $\operatorname{supp}(s)$, is the set $\left\{e_{i} \otimes e_{j} \mid a_{i j} \neq 0\right\}$, and $a_{i j}$ is called the coefficient of $e_{i} \otimes e_{j}$ in $s$.

Definition 3. Given an integer $1 \leq r \leq n$ and a vector $t=\left(t_{1}, \ldots, t_{n}\right)$. We define $v_{i, r}(t)=e_{i}-\tau_{i, r}(t)\left(e_{i}\right)=\left(I-\tau_{i, r}(t)\right)\left(e_{i}\right)$. In other words, we have the following:

For $1 \leq i \leq r-1$, we have $v_{i, r}(t)=$

$(1-t_{1}, \ldots, 1-t_{i-1}, \underbrace{1-t_{i} t_{r}}_{i}, t_{r}\left(1-t_{i+1}\right), \ldots, t_{r}\left(1-t_{r-1}\right), \underbrace{t_{r+1}-1}_{r}, t_{r+2}-1, \ldots, t_{n}-1)^{T}$.

and for $n \geq i>r$, we have $v_{i, r}(t)=$

$(t_{r}\left(t_{1}-1\right), \ldots, t_{r}\left(t_{r-1}-1\right), \underbrace{1-t_{r+1}, \ldots, 1-t_{i}}_{i-r}, 1-t_{i+1} t_{r}, t_{r}\left(1-t_{i+2}\right), \ldots, t_{r}\left(1-t_{n}\right))^{T}$.

Next, we state a lemma similar to that used in [1].

Lemma 1. For $t=\left(t_{1}, \ldots, t_{n}\right)$, we have
(1) $\tau_{i, r}(t)\left(v_{i, s}(t)\right)=v_{i, s}+\left(t_{i} t_{s}-1\right) v_{i, r}(t)$
for $1 \leq i \leq s-1$,
$\tau_{i, r}(t)\left(v_{i, s}(t)\right)=v_{i, s}+\left(t_{i+1} t_{s}-1\right) v_{i, r}(t)$
for $1 \leq s<i$,
(2) $\tau_{i, r}(t)\left(v_{j, s}(t)\right)=v_{j, s}(t)+\left(t_{i}-1\right) v_{i, r}(t)$
for $i<j<s$,
$\tau_{i, r}(t)\left(v_{j, s}(t)\right)=v_{j, s}(t)+t_{s}\left(t_{i}-1\right) v_{i, r}(t)$
for $j<i<s$
$\tau_{i, r}(t)\left(v_{j, s}(t)\right)=v_{j, s}(t)+\left(1-t_{i+1}\right) v_{i, r}(t)$
for $j<s<i$,
(3) $\tau_{i, r}(t)\left(v_{j, s}(t)\right)=v_{j, s}(t)+t_{s}\left(1-t_{i}\right) v_{i, r}(t) \quad$ for $i<s<j$,

$$
\begin{aligned}
& \tau_{i, r}(t)\left(v_{j, s}(t)\right)=v_{j, s}(t)+\left(t_{i+1}-1\right) v_{i, r}(t) \quad \text { for } \quad s<i<j, \\
& \tau_{i, r}(t)\left(v_{j, s}(t)\right)=v_{j, s}(t)+t_{s}\left(t_{i+1}-1\right) v_{i, r}(t) \quad \text { for } \quad s<j<i .
\end{aligned}
$$


For a fixed value of $r$, we use Lemma 1 to determine elements in the group algbera $\mathbb{C}\left(P_{n}\right)$ over $\mathbb{C}$ that send the vector $v_{i, r}$ to the vector $v_{i+1, r}$ and other elements that send the vector $v_{i, r}$ to $v_{i-1, r}$.

Definition 4. Given an integer $r$ such that $1 \leq r \leq n$. Consider the following elements of the pure braid group algebra.

$$
f_{i, r}=\left\{\begin{array}{l}
\tau_{i, r}-\left(t_{i} t_{r}\right) \tau_{i+1, r}, \quad 1 \leq i<r-1 \\
\tau_{i, r}-\left(t_{i} t_{r}\right) \tau_{i+2, r}, \quad i=r-1 \\
\tau_{i, r}-\left(t_{i+1} t_{r}\right) \tau_{i+1, r}, \quad 1 \leq r<i \leq n-1
\end{array}\right.
$$

and

$$
g_{i, r}=\left\{\begin{array}{l}
\tau_{i, r}-\left(t_{i} t_{r}\right) \tau_{i-1, r}, \quad 1 \leq i \leq r-1 \\
\tau_{i, r}-\left(t_{i+1} t_{r}\right) \tau_{i-2, r}, \quad i=r+1 \leq n-1 \\
\tau_{i, r}-\left(t_{i+1} t_{r}\right) \tau_{i-1, r}, \quad r+1<i \leq n-1
\end{array}\right.
$$

Then we have the following lemma.

Lemma 2. Fix an integer $1 \leq r \leq n$. For all integers $1 \leq i \leq n-1$, the action of the elements of the pure braid group algebra, namely, $f_{i, r}$ and $g_{i, r}$, on the vectors $v_{i, r}$ is given by

(i) $f_{i, r}\left(v_{i, r}\right)=\left\{\begin{array}{l}-t_{i} t_{r}{ }^{2}\left(t_{i+1}-1\right) v_{i+1, r}, \quad 1 \leq i<r-1 \\ -t_{r-1} t_{r}\left(1-t_{r+2}\right) v_{r+1, r}, \quad i=r-1 \leq n-3 \\ -t_{i+1} t_{r}^{2}\left(t_{i+2}-1\right) v_{i+1, r}, 1 \leq r<i \leq n-2\end{array}\right.$

and

(ii) $g_{i, r}\left(v_{i, r}\right)=\left\{\begin{array}{l}-t_{i} t_{r}\left(t_{i-1}-1\right) v_{i-1, r}, 1 \leq i \leq r-1 \\ -t_{r+2} t_{r}^{2}\left(1-t_{r-1}\right) v_{r-1, r}, \quad i=r+1 \leq n-1 \\ -t_{i+1} t_{r}\left(t_{i}-1\right) v_{i-1, r}, r+1<i \leq n-1 .\end{array}\right.$

Notation 2. Let $G_{n}\left(x_{1}, \ldots, x_{n}\right)$ denote the reduced Gassner representation of $P_{n}$ under the specialization $t_{i} \rightarrow x_{i}$, where $x_{i}$ is a non-zero complex number.

Lemma 3. Having $U_{r}$ a free normal subgroup of the pure braid group, we let $G_{n}\left(x_{1}, \ldots\right.$, $\left.x_{n}\right): U_{r} \rightarrow G L\left(\mathbb{C}^{n-1}\right)$ be a specialization of the reduced Gassner representation restricted to $U_{r}$ making $\mathbb{C}^{n-1}$ into a $U_{r}$-module, where $n \geq 3$. Then

(a) Let $\mathcal{A}$ be the kernel of the homomorphism $\mathbb{C}\left[U_{r}\right] \rightarrow \mathbb{C}$ induced by $\tau_{i, r} \rightarrow 1$ (the augmentation ideal). Let $x$ be the vector $\left(x_{1}, \ldots, x_{n}\right)$. Then $\mathcal{A} \mathbb{C}^{n-1}$ is equal to the $\mathbb{C}$-vector space spanned by $v_{1, r}(x), \ldots, v_{r-1, r}(x), v_{r+1, r}, \ldots, v_{n, r}$.

(b) If $M$ is a nonzero $U_{r}$-submodule of $\mathbb{C}^{n-1}$, then $\mathcal{A} \mathbb{C}^{n-1} \subseteq M$. Hence $\mathcal{A} \mathbb{C}^{n-1}$ is the unique minimal nonzero $U_{r}$-submodule of $\mathbb{C}^{n-1}$. 
(c) If $p\left(x_{1}, \ldots, x_{n}\right)=\left(x_{r}-1\right)^{n-2}\left(x_{1} x_{2} \ldots x_{n}-1\right) \neq 0$, then $\mathcal{A} \mathbb{C}^{n-1}=\mathbb{C}^{n-1}$, and $G_{n}\left(x_{1}, x_{2}, \ldots, x_{n}\right)$ restricted to $U_{r}$ is irreducible.

Proof. The proof is similar to that in [2]. Here, we will take the free normal subgroup, $U_{r}$, of rank $n-1$. Notice that, in the proof of (b), we need the fact that if $v_{j, r} \in M$ for some $j$ and $r$ then all $v_{i, r} \in M$. This is due to Lemma 1. As for (c), the determinant of the matrix, whose columns are the vectors $v_{1, r}(x), \ldots, v_{n, r}(x)$, is $p(x)=\left(x_{r}-1\right)^{n-2}\left(x_{1} x_{2} \ldots x_{n}-1\right)$, so if $p(x) \neq 0$ then $v_{1, r}(x), \ldots v_{n, r}(x)$ is a basis for $\mathbb{C}^{n-1}$ and $\mathcal{A} \mathbb{C}^{n-1}=\mathbb{C}^{n-1}$. For more details, see [4].

Hence, $\mathcal{A} \mathbb{C}^{n-1}$ is its unique minimal nonzero $U_{r}$-submodule. Of course $\mathcal{A} \mathbb{C}^{n-1}=$ $\mathbb{C}^{n-1}$ when $G_{n}\left(x_{1}, \ldots, x_{n}\right)$ is irreducible. A result in [1] states that if for some $i$ and $j(i<$ j) $x_{i} x_{j} \neq y_{i} y_{j}$ and $x_{\alpha} x_{j} y_{\alpha} y_{j} \neq 1$ for every $\alpha \in\{1, \ldots, j-1\}$ and $x_{\alpha+1} x_{j} y_{\alpha+1} y_{j} \neq$ 1 for every $\alpha \in\{j, \ldots, n-1\}$ then $G_{n}(x) \otimes G_{n}(y): P_{n} \rightarrow G L\left(\mathbb{C}^{n-1} \otimes \mathbb{C}^{n-1}\right)$ is irreducible (see [1]). In our work, we improve the result by replacing the conditions above by the conditions $x_{i} x_{j} \neq y_{i} y_{j}$ for some $i \neq j$ and $x_{\alpha} x_{k} y_{\alpha} y_{k} \neq 1$ for some $\alpha$ and $k(\alpha \neq k)$.

\section{Claims in [1] and a summary of their proofs}

Before we proceed in proving Proposition 1, we state the main results obtained in our previous work [1]. The notations used here are the same as in Section 1.

Let $M$ be a non zero $P_{n^{-}}$submodule of $\mathbb{C}^{n-1} \otimes \mathbb{C}^{n-1}$, when $n \geq 3$. First, we observe that if $e_{u} \otimes e_{v} \in \operatorname{supp}(m)$ for some $m \in M$ then $e_{\alpha} \otimes e_{v} \in \operatorname{supp}\left(\tau_{u, r}\left(e_{u} \otimes e_{v}\right)\right)$ for every choice of $\alpha=1, \ldots, n-1$ and $v \neq u$. This is clear because of our assumption that none of the parameters $t_{i}$ 's is equal to zero or one.

(1) Claim 1. If $x_{i} x_{j} \neq y_{i} y_{j}$ for some $i$ and $j$ then there exists an $s \in\{1, \ldots, n-1\}$ such that $e_{s} \otimes e_{s} \in \operatorname{supp}(m)$ for some $m \in M$ and $s \in\{1, \ldots, n-1\}$.

Proof of Claim 1. Here, we may take $r$ to be the given integer $j$.

Case 1: Suppose that there exists an $s$ and $m \in M$ such that $e_{s} \otimes e_{s} \in \operatorname{supp}(m)$, then we are done.

Case 2: Suppose that there exists $(s, t)$ with $1 \leq s, t \leq n-1$ and $s \neq t$ such that

$$
m=a\left(e_{s} \otimes e_{t}\right)+W,
$$

where $a \in \mathbb{C}^{*}$ and $\operatorname{supp}(W)$ does not contain $e_{s} \otimes e_{t}, e_{t} \otimes e_{s}$. We also assume that $\operatorname{supp}(W)$ does not contain $e_{\alpha} \otimes e_{\alpha}$ for any $\alpha$.

Then $\tau_{t, j}(m)=a\left(e_{s} \otimes e_{t}-v_{t, j}\right)+\tau_{t, j}(W)$, which implies that $e_{s} \otimes e_{s} \in \operatorname{supp}\left(\tau_{t, j}(m)\right)$ and so we are done.

Case 3: Suppose that for any pair $(s, t)$ and any $m \in M$ such that $e_{s} \otimes e_{t} \in$ $\operatorname{supp}(m)$, we have that $e_{t} \otimes e_{s} \in \operatorname{supp}(m)$ as well. That is, consider $m \in M$ such that

$$
m=a\left(e_{s} \otimes e_{t}\right)+b\left(e_{t} \otimes e_{s}\right)+W, \quad \text { where }
$$


$\operatorname{supp}(W)$ does not contain $e_{s} \otimes e_{t}, e_{t} \otimes e_{s}$ and $e_{\alpha} \otimes e_{\alpha}$ for any $\alpha$. In this case, $W$ is either zero or its elements are of the form $\sum_{k, l}\left(c_{k, l} e_{k} \otimes e_{l}+d_{l, k} e_{l} \otimes e_{k}\right)$.

Here the constants $a, b, c_{k, l}, d_{l, k} \in \mathbb{C}^{*}$.

Applying $\tau_{t, j}$, we observe that $e_{i} \in \operatorname{supp}\left(\tau_{t, j}\left(e_{t}\right)\right)$, where $i$ is the integer given by the hypothesis of Proposition 1. Then

$$
\tau_{t, j}(m)=a\left(e_{s} \otimes e_{i}\right)+b\left(e_{i} \otimes e_{s}\right)+W, \quad \text { where }
$$

$\operatorname{supp}(W)$ does not contain $e_{s} \otimes e_{i}, e_{i} \otimes e_{s}$, and both of $a, b$ are not zeros. For simplicity, we denote $\tau_{t, j}(m)$ by $m$.

If $e_{\alpha} \otimes e_{\alpha} \in \operatorname{supp}(W)$ for some $\alpha$, then we are done. If not, we see that

$$
\begin{gathered}
a M+b N=\text { coefficient of } e_{s} \otimes e_{s} \text { in } \tau_{i, j}(m) \text { and } \\
a M\left(1+y_{i} y_{j}\right)+b N\left(1+x_{i} x_{j}\right)=\text { coefficient of } e_{s} \otimes e_{s} \text { in }\left(\tau_{i, j}\right)^{2}(m) .
\end{gathered}
$$

The values of $M$ and $N$ are not zeros and can be obtained directly from Definition 3 . The determinant

$$
\operatorname{det}\left(\begin{array}{cc}
M & N \\
M\left(1+y_{i} y_{j}\right) & N\left(1+x_{i} x_{j}\right)
\end{array}\right)=M N\left(x_{i} x_{j}-y_{i} y_{j}\right)
$$

is nonzero, since $x_{i} x_{j}-y_{i} y_{j} \neq 0$ by hypothesis. Then one of $\tau_{i, j}(m),\left(\tau_{i, j}\right)^{2}(m)$ has $e_{s} \otimes e_{s}$ in its support.

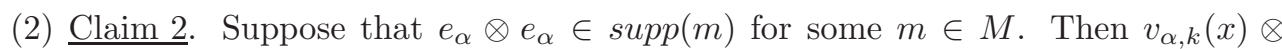
$v_{\alpha, k}(y) \in M$ if $x_{\alpha} x_{k} y_{\alpha} y_{k} \neq 1$ for $\alpha=1, \ldots, k-1$ and $x_{\alpha+1} x_{k} y_{\alpha+1} y_{k} \neq 1$ for $\alpha=$ $k, \ldots, n-1$.

Proof of Claim 2. A calculation shows that

$\left(\tau_{\alpha, k}-1\right)\left(\tau_{\alpha, k}-\gamma_{\alpha} y_{k}\right)\left(\tau_{\alpha, k}-\beta_{\alpha} x_{k}\right)\left(e_{\alpha} \otimes e_{\alpha}\right)=\gamma_{\alpha} \beta_{\alpha} x_{k} y_{k}\left(\beta_{\alpha} x_{k} \gamma_{\alpha} y_{k}-1\right)\left(v_{\alpha, k}(x) \otimes v_{\alpha, k}(y)\right)$

and

$$
\left(\tau_{\alpha, k}-1\right)\left(\tau_{\alpha, k}-\gamma_{\alpha} y_{k}\right)\left(\tau_{\alpha, k}-\beta_{\alpha} x_{k}\right)\left(e_{u} \otimes e_{v}\right)=0 \text { if }(u, v) \neq(\alpha, \alpha) .
$$

Here, we have

$$
\beta_{\alpha}=x_{\alpha}, \quad \gamma_{\alpha}=y_{\alpha} \text { for } \alpha=1, \ldots, k-1
$$

and

$$
\beta_{\alpha}=x_{\alpha+1}, \quad \gamma_{\alpha}=y_{\alpha+1} \text { for } \alpha=k, \ldots, n-1 \text {. }
$$

\section{Main Theorem}

To prove our main theorem, Theorem 1, we introduce Proposition 1 and Lemma 4. 
Proposition 1. Suppose that $x=\left(x_{1}, \ldots, x_{n}\right)$ and $y=\left(y_{1}, \ldots, y_{n}\right) \in \mathbb{C}^{n}$, where $x_{s}, y_{s} \in$ $\mathbb{C}-\{0,1\}$ for $1 \leq s \leq n$. Suppose also that $x_{i} x_{j} \neq y_{i} y_{j}$ for some $i \neq j$ and $x_{\alpha} x_{k} y_{\alpha} y_{k} \neq$ 1 for some $\alpha \neq k$. Let $M$ be a nonzero $P_{n}$-submodule of $\mathbb{C}^{n-1} \otimes \mathbb{C}^{n-1}$ under the action of $G_{n}(x) \otimes G_{n}(y): P_{n} \rightarrow G L\left(\mathbb{C}^{n-1} \otimes \mathbb{C}^{n-1}\right)$, where $n \geq 3$. Then $M$ contains all $v_{p, j}(x) \otimes v_{q, j}(y)$ for $p, q \in\{1, \ldots, j-1, j+1, \ldots, n\}$. Thus $M$ contains $\mathcal{A} \mathbb{C}^{n-1} \otimes \mathcal{A} \mathbb{C}^{n-1}$, where the action of $P_{n}$ on the first factor is induced by $G_{n}\left(x_{1}, \ldots, x_{n}\right)$ and the action of $P_{n}$ on the second factor is induced by $G_{n}\left(y_{1}, \ldots, y_{n}\right)$.

Proof. We will show that once there exists a vector $v_{a, k} \otimes v_{a, k} \in M$ for some $a$ then all other vectors $v_{l, k} \otimes v_{l, k}$ will also be in $M$ using the hypothesis of our proposition and Lemma 2. Here $k$ is the integer given by the proposition and $l \in\{1, \ldots, k-1, k+1, \ldots, n\}$.

By Claim 1 of $[1, \mathrm{p} .14]$, we have that $e_{s} \otimes e_{s} \in \operatorname{supp}(m)$ for some $s$. This is due to the fact that $x_{i} x_{j} \neq y_{i} y_{j}$ for some $i$ and $j$. Having that $e_{s} \otimes e_{s} \in \operatorname{supp}(m)$ for some $m \in M$, we write $m=\alpha_{s} e_{s} \otimes e_{s}+W$ and $\operatorname{supp}(W)$ does not contain $e_{s} \otimes e_{s}$. Here $\alpha_{s} \in \mathbb{C}^{*}$. It follows that

$$
\begin{aligned}
\tau_{s, k}(m) & =\alpha_{s} \tau_{s . k}\left(e_{s} \otimes e_{s}\right)+\tau_{s, k}(W) \\
& =\alpha_{s}\left(e_{s}-v_{s, k} \otimes e_{s}-v_{s, k}\right)+\tau_{s, k}(W) \\
& =\alpha_{s}\left(\sum_{l=1}^{n-1} A_{l} e_{l} \otimes \sum_{l=1}^{n-1} B_{l} e_{l}\right)+\tau_{s, k}(W) .
\end{aligned}
$$

This implies that $e_{l} \otimes e_{l} \in \operatorname{supp}\left(\tau_{s, k}(m)\right)$ for every $l \in\{1, \ldots, n-1\}$. In particular, let $l=\alpha$ such that $x_{\alpha} x_{k} y_{\alpha} y_{k} \neq 1$. Then we get, by Claim 2 of [1, p.14], that

$$
v_{\alpha, k} \otimes v_{\alpha, k} \in M
$$

Applying Lemma 2, we have that $f_{\alpha, k}\left(v_{\alpha, k} \otimes v_{\alpha, k}\right) \in M$, which implies that $v_{\alpha+1, k} \otimes$ $v_{\alpha+1, k} \in M$. Similarly, we also have that $g_{\alpha, k}\left(v_{\alpha, k} \otimes v_{\alpha, k}\right) \in M$, which implies that $v_{\alpha-1, k} \otimes v_{\alpha-1, k} \in M$. After a consecuitive use of $f_{\alpha, k}, f_{\alpha+1, k}, \ldots$ and $g_{\alpha, k}, g_{\alpha-1, k}, \ldots$, we obtain that

$$
v_{l, k} \otimes v_{l, k} \in M \text { for every } l \in\{1, \ldots, k-1, k+1, \ldots, n\} \text {. }
$$

As in Lemma 2, for that integer $k$ and any integer $l$, we find elements in the group algebra $\mathbb{C}\left(P_{n}\right)$ over $\mathbb{C}$ that send the vectors $v_{l, k}$ to the vectors $v_{l, k+1}$ when $1 \leq k<n$, and other elements in $\mathbb{C}\left(P_{n}\right)$ that send the vectors $v_{l, k}$ to the vectors $v_{l, k-1}$. For example, we can consider the element

$$
\gamma_{l, k}=\tau_{l-1, k}-\left(t_{l-1} t_{k}\right) \tau_{l, k+1} \quad \text { when } \quad l \leq k .
$$

Here, $k$ is the integer given in proposition 1 and $l$ is any integer in $\{1, \ldots, k-1, k+$ $1, n\}$. We have $\gamma_{l, k}\left(v_{l-1, k}\right)=-t_{l-1} t_{k}^{2}\left(t_{l}-1\right) v_{l, k+1}$.

Since $v_{l-1, k} \otimes v_{l-1, k} \in M$ for every value of $l$, it follows that $v_{l, k+1} \otimes v_{l, k+1} \in M \quad(1 \leq$ $k<n)$. Now apply $g_{l, k+1}$ from Lemma 2, we obtain that $v_{l-1, k+1} \otimes v_{l-1, k+1} \in M$ for every value of $l$. 
Similarly, we find other elements in $\mathbb{C}\left(P_{n}\right)$ to show that given any integer $l \in\{1, \ldots, k-$ $1, k+1, n\}$ such that $v_{l-1, k} \otimes v_{l-1, k} \in M$, we obtain that $v_{l-1, k-1} \otimes v_{l-1, k-1} \in M$. Therefore, by direct computations, and for all integers $p$ and $t$, we get

$$
v_{p, t} \otimes v_{p, t} \in M \text {. }
$$

In particular, let $t=j$. Then we have that $v_{p, j} \otimes v_{p, j} \in M$. Given integers $p, q \in$ $\{1, \ldots, j-1, j+1, \ldots, n\}$ and $p \neq q$. We will show that $v_{p, j} \otimes v_{q, j} \in M$. We consider two cases.

Case 1: Let $p=i$. ( $i$ and $j$ are the integers given by the hypothesis of Proposition 1). By our assumption, we have that $q \neq i$. By applying $\tau_{q, j}$ on $\left(v_{i, j} \otimes v_{i, j}\right)$, we obtain

$$
a v_{q, j} \otimes v_{i, j}+b v_{i, j} \otimes v_{q, j} \in M \quad(a \neq 0, b \neq 0) .
$$

Applying $\tau_{i, j}$, we obtain

$$
a y_{i} y_{j} v_{q, j} \otimes v_{i, j}+b x_{i} x_{j} v_{i, j} \otimes v_{q, j} \in M .
$$

Combining (3) and (4), we get

$$
b\left(y_{i} y_{j}-x_{i} x_{j}\right) v_{i, j} \otimes v_{q, j} \in M
$$

Since $x_{i} x_{j} \neq y_{i} y_{j}$, it follows that

$$
v_{i, j} \otimes v_{q, j} \in M \text {. }
$$

Case 2: Let $p \neq i, q \neq i$ and $p \neq q$. By applying $\tau_{i, j}$ on $\left(v_{p, j} \otimes v_{p, j}\right)$, we obtain

$$
a v_{i, j} \otimes v_{p, j}+b v_{p, j} \otimes v_{i, j} \in M \quad(a \neq 0, b \neq 0) .
$$

Applying $\tau_{i, j}$ again, we obtain

$$
a x_{i} x_{j} v_{i, j} \otimes v_{p, j}+b y_{i} y_{j} v_{p, j} \otimes v_{i, j} \in M .
$$

Combining (5) and (6), we get that

$$
v_{p, j} \otimes v_{i, j} \in M
$$

Now apply $\tau_{q, j}$, we get that for $p, q \in\{1, \ldots, j-1, j+1, \ldots, n\}$

$$
v_{p, j} \otimes v_{q, j} \in M \text {. }
$$

In order to simplify the conditions in Proposition 1 further, we prove the next Lemma.

Lemma 4. Given $n \geq 3$ and non-zero complex numbers $x_{1}, \ldots, x_{n}$ and $y_{1}, \ldots, y_{n}$. Then the following holds.

(i) If $x_{v} \neq \pm y_{v}$ for some $v$ then $x_{a} x_{b} \neq y_{a} y_{b}$ for some distinct $a, b$.

(ii) If $x_{u} \neq \pm y_{u}{ }^{-1}$ for some $u$ then $x_{g} x_{h} y_{g} y_{h} \neq 1$ for some distinct $g, h$. 
Proof. (i) Assume, to get contradiction, that $x_{a} x_{b}=y_{a} y_{b}$ for $a \neq b$. Then, we have that

$$
\begin{gathered}
x_{1} x_{n}=y_{1} y_{n}, \\
\vdots \\
x_{n-1} x_{n}=y_{n-1} y_{n} .
\end{gathered}
$$

By solving the above equations, we get that $x_{u} y_{v}=x_{v} y_{u}$, where $1 \leq u<v \leq$ $n-1$. Since we also have that $x_{u} x_{v}=y_{u} y_{v}$, it follows that $x_{v}= \pm y_{v}$ for every $v \in\{1, \ldots, n\}$, a contradiction.

(ii) Assume, to get contradiction, that $x_{g} x_{h} y_{g} y_{h}=1$ for $g \neq h$. Then, we have that

$$
\begin{gathered}
x_{1} x_{n} y_{1} y_{n}=1, \\
\vdots \\
x_{n-1} x_{n} y_{n-1} y_{n}=1 .
\end{gathered}
$$

This implies that $x_{u} y_{u}=x_{u+1} y_{u+1}$, where $u \in\{1, \ldots, n-2\}$. Having that $x_{u} x_{u+1} y_{u} y_{u+1}=1$, it follows that $x_{u}= \pm y_{u}{ }^{-1}$ for every $u \in\{1, \ldots, n\}$, a contradiction.

Consider the representation $G_{n}\left(t_{1}, \ldots, t_{n}\right): P_{n} \rightarrow G L_{n-1}\left(\mathbb{C}\left[t_{1}^{ \pm 1}, \ldots, t_{n}^{ \pm 1}\right]\right)$, where $t_{1}, \ldots, t_{n}$ are indeterminates. Specializing $t_{1}, \ldots, t_{n}$ to nonzero complex numbers $x_{1}, \ldots, x_{n}$ defines a representation $G_{n}\left(x_{1}, \ldots, x_{n}\right): P_{n} \rightarrow G L_{n-1}(\mathbb{C})=G L\left(\mathbb{C}^{n-1}\right)$ which is irreducible if and only if $q\left(x_{1}, \ldots, x_{n}\right)=x_{1} \ldots x_{n}-1 \neq 0$. (see [1] and [4]).

By combining Proposition 1 and Lemma 4, we get our main theorem.

Theorem 1. For $n \geq 3$, consider the tensor product of irreducible representations $G_{n}\left(x_{1}, \ldots, x_{n}\right) \otimes G_{n}\left(y_{1}, \ldots, y_{n}\right): P_{n} \rightarrow G L\left(\mathbb{C}^{n-1} \otimes \mathbb{C}^{n-1}\right)$, where $q\left(x_{1}, \ldots, x_{n}\right) \neq$ 0 and $q\left(y_{1}, \ldots, y_{n}\right) \neq 0$. If for some $i$ and $j, \quad x_{i} \neq \pm y_{i}$ and $x_{j} \neq \pm y_{j}{ }^{-1}$ then the above representation is irreducible.

Proof. By Lemma 4 and Proposition 1, we have that $\mathcal{A} \mathbb{C}^{n-1} \otimes \mathcal{A} \mathbb{C}^{n-1}$ is the unique minimal nonzero $P_{n}$-submodule of $\mathbb{C}^{n-1} \otimes \mathbb{C}^{n-1}$. In particular, it is an irreducible $P_{n^{-}}$ module. The fact that $q\left(x_{1}, \ldots, x_{n}\right) \neq 0$ and $q\left(y_{1}, \ldots, y_{n}\right) \neq 0$ implies that the left factor $\mathcal{A} \mathbb{C}^{n-1}$ corresponds to the representation $G_{n}\left(x_{1}, \ldots, x_{n}\right)$ and the right factor $\mathcal{A} \mathbb{C}^{n-1}$ corresponds to the representation $G_{n}\left(y_{1}, \ldots, y_{n}\right)$.

\section{References}

[1] M. Abdulrahim, Tensor products of the Gassner representation of the pure braid group, Open Math J., 2 (2009), 12-15.

[2] M. Abdulrahim and E. Formanek, Tensor products of specializations of the Burau representation. J. Pure Appl. Algebra 203, no. 1-3 (2005), 104-112. 
[3] J. S. Birman, Braids, Links and Mapping Class Groups, Annals of Mathematical Studies 82, Princeton University Press, New Jersey, 1975.

[4] E. Formanek, Braid group representations of low degree, Proc. London Math. Soc., 73 (1996), 279-322.

Department of Mathematics, Beirut Arab University, P.O. Box 11-5020, Beirut, Lebanon.

E-mail: mna@bau.edu.lb 